\title{
Clinical Pharmacology of Intravenous Paracetamol in Neonates
}

\author{
Karel Allegaert ${ }^{*}, 1$, Marc van de Velde ${ }^{2}$ and Gunnar Naulaers ${ }^{1}$ \\ ${ }^{I}$ Neonatal Intensive Care Unit and ${ }^{2}$ Department of Anaesthesiology, University Hospitals Leuven, Herestraat 49, 3000 \\ Leuven, Belgium
}

\begin{abstract}
Effective analgesia in neonates is still hampered due to the lack of data on pharmacokinetics and -dynamics of analgesics in this specific population. To a certain extent, this is even true for paracetamol. An intravenous formulation might improve prediction of concentration and consequent effect compared to enteral administration. In this review, we summarize the consecutive steps reported in literature to document the pharmacokinetics, metabolism and hepatic tolerance of intravenous paracetamol in neonates.

Based on a single intravenous dose study, pharmacokinetics estimates were calculated and a repeated dose regimen was developed. This dose regimen was subsequently evaluated in a repeated dose study, and paracetamol metabolism was described during repeated dose administration. Finally, hepatic tolerance in neonates was documented.

Based on the available pharmacokinetic estimates, it seems that intravenous paracetamol is an attractive analgesic to be used in neonates, as an alternative or add-on therapy for opioid administration. After the documentation of the pharmacokinetics of paracetamol in neonates and in the absence of any data on pharmacodynamics of intravenous paracetamol in this population, prospective, well designed and appropriated powered pharmacodynamic studies in neonates are urgently needed.
\end{abstract}

\section{INTRODUCTION}

Adequate management of pain in neonates is a major issue, not only from an ethical perspective, but also to improve short and long term outcome [1]. Effective treatment of pain in this population is still in part hampered due to the limited volume of data on the pharmacokinetics and dynamics of analgesics prescribed. To a certain extent, this is even true for paracetamol [2]. Paracetamol, N-acetyl-paminophenol, is a readily available antipyretic and analgesic agent. It is the most often prescribed drug for treatment of mild to moderate pain or fever in infants, including neonates and can be administered by oral, rectal but also by intravenous route [3]. Propacetamol (ProDafalgan ${ }^{\circledR}$, Bristol-Myers Squibb, Brain-l'Alleud, Belgium) is a pro-drug of paracetamol and is hydrolysed by plasma esterase after intravenous administration such that $1 \mathrm{~g}$ of propacetamol is hydrolysed to $0.5 \mathrm{~g}$ of paracetamol. A new formulation of iv paracetamol (Perfusalgan ${ }^{\circledR}$, Bristol-Myers Squibb, Brain-l'Alleud, Belgium) became more recently available [4]. The use of an intravenous administration might improve prediction of concentration and consequent effect compared to rectal and/or oral formulations by the elimination of plasma variability due to absorption kinetics and bio-availability. In addition, an intravenous route enables administration of paracetamol when the enteral route is not (yet) accessible [5].

In adults, paracetamol is almost exclusively eliminated by renal way after conjugation with either glucuronic acid to paracetamol-glucuronide (APAP-G, 47-62\%) or with sulfate

*Address correspondence to this author at the Neonatal Intensive Care Unit, Division of Woman and Child, University Hospitals Leuven, campus Gasthuisberg, Herestraat 49, 3000 Leuven, Belgium; Tel: 00-32-16-343210; Fax: 00-32-16-343209; E-mail: karel.allegaert@uz.kuleuven.ac.be to paracetamol sulfate (APAP-S, 25-36\%) resulting in a APAP-G/APAP-S ratio of about 2 . Only limited amounts are excreted in the urine as free paracetamol or are metabolized through oxidation to N-acetyl-p-benzoquinone-imine (NAPQI) and into 3-hydroxy-APAP by the cytochrome $\mathrm{p}$ 450 enzyme system. If not depleted, glutathione conjugates with the hepatotoxic metabolite NAPQI resulting in cysteine and mercapturic acid metabolites (Fig. 1). Uridinediphosphate-glucuronosyltransferases (UGT's) are part of a superfamily of enzymes that catalyse the addition of a glycosyl group from a nucleotide sugar to a small molecule enhancing renal elimination of this molecule. Paracetamol is mainly metabolized by UGT-1A6 and to a much lesser extent by UGT-1A1 and UGT-1A9. This iso-enzyme specific activity depends at least in part on the concentration of the drug. UGT-1A6 activity has been observed in hepatic and renal tubular cells [6]. A maturational trend with a progressive increase in APAP-G elimination has been described in several single dose enteral paracetamol studies in neonates, infants and children, based on a progressive increase in activity of the glucuronidation pathway during childhood only reaching an adult $\mathrm{G} / \mathrm{S}$ ratio at the age of 8 to 10 year $[7,8]$. Data available on paracetamol metabolism in neonates were until recently limited to single dose administration and no data on safety assessment in neonates were available.

Anderson et al. illustrated in children that the pharmacokinetics of paracetamol in cerebrospinal fluid (CSF) reflect more the effect compartment compared to serum pharmacokinetics. After enteral administration, peak serum concentrations were reached after 90 to $120 \mathrm{~min}$. However, an additional delay of one hour between peak serum concentration and maximal analgesia was observed. This clinical observation suggests that paracetamol acts at least in part by central nervous system mediated processes [9]. The delay 


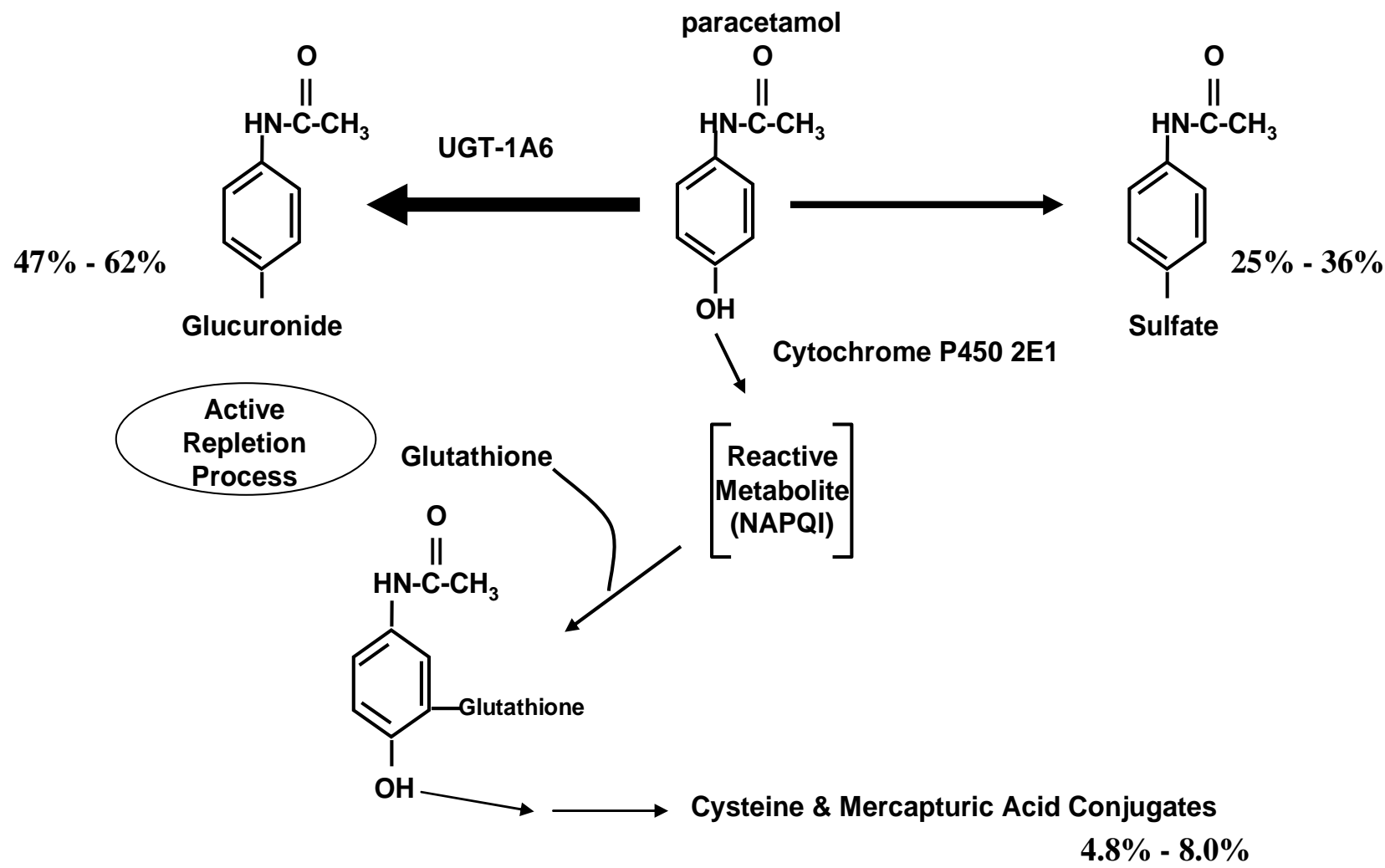

Fig. (1). Paracetamol metabolism.

between peak concentration of paracetamol in CSF and peak serum concentration has been observed in a population approach in adults after intravenous administration and reflects the blood-brain barrier for paracetamol in adults. Observations on the CSF kinetics of paractamol and its blood-brain barrier in neonates and infants were recently published [10, 11].

In this review, we would like to summarize our stepwise approach to describe intravenous paracetamol disposition, metabolism and hepatic tolerance (pharmacokinetics) in preterm and term neonates $[4,5,12,13]$. In contrast to the available observations on the pharmacokinetics of this drug in neonates, we are unaware of any pharmacodynamic study (level of analgesia) on intravenous paracetamol in neonates. Taking the potential side-effects of opioids in this specific population (paralytic ileus, bladder paralysis, hypoventilation) into account, prospective, well designed and appropriated powered studies are urgently needed [1].

\section{PHARMACOKINETICS OF INTRAVENOUS PARACETAMOL IN NEONATES: EFFECT OF THE POSTMENSTRUAL AGE}

Neonates admitted within the first $24 \mathrm{~h}$ of life and with an arterial line in place were considered for inclusion if propacetamol was administered. The decision to prescribe propacetamol or any other analgesic was made by the attending neonatologist. Propacetamol was administered when infants underwent minor, painful procedures (e.g. insertion of peripheral arterial or venous line, insertion of central venous line or placement of a chest tube) or as additional therapy in infants on opioids. Exclusion criteria were major congenital malformations or severe birth asphyxia (Apgar $<4$ at
$5 \mathrm{~min}$ ). The initial dose, $20 \mathrm{mg}$ propacetamol (equal to 10 $\mathrm{mg}$ paracetamol)/kg dose was extrapolated from data in literature, with the intention to adapt this dose if interim analysis of paracetamol levels in the first 15 infants were suboptimal or toxic, defined by serum levels below $5 \mathrm{mg} / \mathrm{l}$ or above $20 \mathrm{mg} / \mathrm{l}$ within 8-10 h after administration. Propacetamol was administered as a $15-\mathrm{min}$ infusion to avoid local discomfort. Blood samples $(0.2 \mathrm{ml})$ were taken from an arterial line $30,60,90,120,180,240$ and $600 \mathrm{~min}$ after initiation of intravenous administration, but the maximal amount of blood collected in a single neonate was $1 \mathrm{ml} / \mathrm{kg}$. After centrifugation, samples were stored at $-20^{\circ} \mathrm{C}$ until analysis. Paracetamol serum concentrations were determined using fluorescence polarization immunoassay (Adx system, Abbott Laboratories, North Chicago IL).

Pharmacokinetics were calculated assuming a linear onecompartment model with instantaneous input and first-order output. For every patient a logarithmic linear trend line was calculated, based on at least three serum samples. Distribution volume $\left(\mathrm{V}_{\mathrm{d}}, 1 / \mathrm{kg}\right)$ and concentration at $\mathrm{t}=0\left(\mathrm{C}_{0}\right)$ were calculated. The slope of the curve was used to calculate the elimination constant $\mathrm{K}(\mathrm{K}=$ slope $\mathrm{x} 2.303)$, terminal elimination half life $(\mathrm{T} 1 / 2=0,693 / \mathrm{K})$ and total clearance $\left(\mathrm{Cl}_{\mathrm{t}}=\mathrm{K}\right.$ $\mathrm{x} \mathrm{V}_{\mathrm{d}}$ ). Results are reported by mean, standard deviation (SD) and range.

Thirty neonates of variable GA were included. Fifteen neonates received a $20 \mathrm{mg}$ and 15 neonates received a $40 \mathrm{mg}$ dose of propacetamol, equivalent to 10 or $20 \mathrm{mg}$ of paracetamol. Mean birth weight was 2111 (SD 1094) g and mean GA was 33.8 (SD 3.9) weeks. Mean postnatal age at inclusion was 12.7 (SD 6.4) h. Ten neonates had a GA $\geq 37$ weeks, 20 were preterm $(<37$ weeks GA), of whom 10 were $<32$ weeks GA. 
No significant difference in $V_{d}(1 / \mathrm{kg})$ between preterm and term infants was documented but $\mathrm{T} 1 / 2$ and $\mathrm{Cl}$ were significantly different. Mean T $1 / 2$ in preterm infants was 277 (SD 143) min. In term infants, it was 172 (SD 59) min. Correlation between GA and $\mathrm{T} 1 / 2(\mathrm{r}=-0.46, \mathrm{p}<0.05$, Fig. (2)) was stronger than between birth weight and $\mathrm{T} 1 / 2(\mathrm{r}=-0.39$, $\mathrm{p}<0.05)$. Mean $\mathrm{Cl}$ was significantly $(\mathrm{p}<0.05)$ lower in preterm infants compared to term $(0.116$ versus $0.170 \mathrm{l} / \mathrm{kg} / \mathrm{h})$ infants. There was still major interindividual variability in the pharmacokinetics of paracetamol not explained by the GA. This variability was neither gender-related, nor associated with the prenatal administration of betamethasone [4].

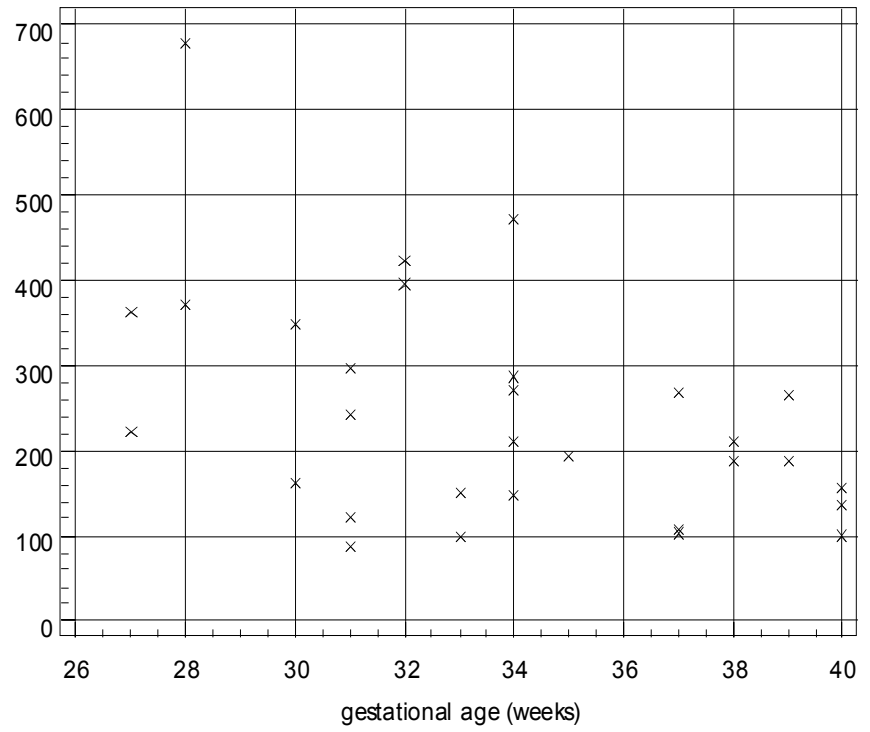

Fig. (2). Serum elimination half life ( $\mathrm{min}$ ) in 30 neonates on day 1 in whom a single dose of propacetamol (either 20 or $40 \mathrm{mg} / \mathrm{kg}$, equal to 10 or $20 \mathrm{mg} / \mathrm{kg}$ of paracetamol) was administered Figure adapted from ref. [4].

Based on these preliminary findings, a repeated dose scheme was developed and subsequently further validated in a repeated dose study (Table 1) $[5,14]$. The loading dose, irrespective of the gestational or postmenstrual age hereby reflects the higher distribution volume of paracetamol com- pared to children or adults, while the age-dependent daily dose reflects the maturation in clearance of paracetamol with increasing postmenstrual age (Fig. 3).

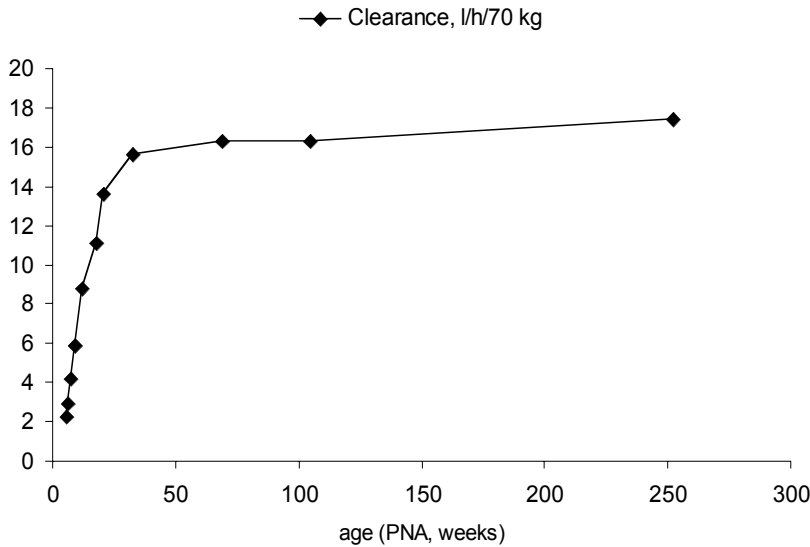

Fig. (3). Paracetamol clearance $(1 / \mathrm{h} / 70 \mathrm{~kg})$ in preterm and term neonates and toddlers up to 5 years of age, equal to 254 weeks after allometric scaling. The progressive increase in clearance is most pronounced in the first year of life.

All concentration-time profiles in term and preterm neonates were hereby entered in a population pharmacokinetic analysis using a non-linear mixed effects model (NONMEM) to describe maturational aspects of pharmacokinetics of intravenous paracetamol in term and preterm neonates. Clearance increased from $2.85 \mathrm{~L} / 70 \mathrm{~kg}, \mathrm{CV} 40.7 \%$ at 27 weeks postmenstrual age to reach $7.05 \mathrm{~L} / \mathrm{h} / 70 \mathrm{~kg}$ by 42 weeks (standardised to a $70 \mathrm{~kg}$ person using allometric " $1 / 4$ power" models). Between occasion variability for volume of distribution and clearance were $17.4 \%$ and $26 \%$ respectively. (warning: propacetamol doses, for intravenous paracetamol $50 \%$ of dose) (Table 1) [5].

\section{METABOLISM AND HEPATIC TOLERANCE DUR- ING REPEATED IV PARACETAMOL ADMINI- STRATION IN NEONATES}

To further evaluate the dosing regimen on aspects of drug metabolism and tolerance in early life, urinary samples were

Table 1. Dosing Suggestions, Based on the Pharmacokinetic Studies Performed in Neonates as used in the Leuven Neonatal Intensive Care Unit [14]

\begin{tabular}{|c|c|c|c|}
\hline \multirow[t]{3}{*}{ Loading Dose: } & \multicolumn{3}{|c|}{ Independent of Postmenstrual Age } \\
\hline & \multicolumn{2}{|c|}{ propacetamol } & $40 \mathrm{mg} / \mathrm{kg}$ iv \\
\hline & \multicolumn{2}{|c|}{ IV paracetamol } & $20 \mathrm{mg} / \mathrm{kg}$ iv \\
\hline Maintenance Dose: & \multicolumn{3}{|c|}{ Dependent of Postmenstrual Age } \\
\hline \multirow[t]{3}{*}{ propacetamol } & $<31$ & PMA & $2 \times 20 \mathrm{mg} / \mathrm{kg} /$ day \\
\hline & $31-36$ & PMA & $3 \times 20 \mathrm{mg} / \mathrm{kg} /$ day \\
\hline & $>36$ & PMA & $4 \times 20 \mathrm{mg} / \mathrm{kg} /$ day \\
\hline \multirow[t]{3}{*}{ IV paracetamol } & $<31$ & PMA & $2 \times 10 \mathrm{mg} / \mathrm{kg} /$ day \\
\hline & $31-36$ & PMA & $3 \times 10 \mathrm{mg} / \mathrm{kg} /$ day \\
\hline & $>36 \quad$ wks & PMA & $4 \times 10 \mathrm{mg} / \mathrm{kg} /$ day \\
\hline
\end{tabular}

In the implementation of the dosing regimes in clinical care, frequency of administration and relative uniformity have also been considered for reasons of safety. These dose suggestions are not equal to the dosing regimes registered by the company. The current regimen has only been evaluated on aspects of pharmacokinetics, metabolism and hepatic tolerance. No data on pharmacodynamics of this or any other iv paracetamol regimen are currently available in neonates. 
collected in neonates in whom repeated intravenous propacetamol was administered as part of their routine clinical care (Table 1 regimen). We hereby evaluated the effect of postmenstrual age, of postnatal age and of repeated administration of paracetamol on observed variability of glucuronidation in neonates [12].

A significant increase in the relative contribution of paracetamol-glucuronide to overall urinary paracetamol elimination (i.e. $\mathrm{G} / \mathrm{T}$ ratio) with increasing postnatal age $(\mathrm{p}<0.0001)$ and postmenstrual age $(\mathrm{p}=0.0055)$ were observed. In addition, an increase $(\mathrm{p}=0.0005)$ in $\mathrm{G} / \mathrm{T}$ ratio was documented during repeated administration and remained a significant variable to explain $\mathrm{G} / \mathrm{T}$ ratio $(\mathrm{p}<0.01)$ in a multiple regression model. The effect of repeated administration of paracetamol on UGT-activity in neonates remained significant using the normal distributed $\mathrm{G} / \mathrm{T}$ ratio in a multiple linear regression model to correct for the effect of postnatal and postmenstrual age [12]. These findings can be explained by 'induction' of enzymatic enzyme activity (UGTisoenzymes). An alternative explanation might be a switch from biliary to renal elimination of glucuronidated metabolites similar to what has only more recently been described in rodents and human adults $[15,16]$.

Paracetamol toxicity in children remains a concern $[17$, 18]. Specific subgroups, with reduced clearance capacity or in whom total parental nutrition is administered - like neonates - might display an increased risk to develop toxicity while an intravenous route will result in a higher peak paracetamol plasma concentrations [19]. We therefore decided to evaluate aspects of hepatic tolerance in pre(term) neonates during repeated dose administration of the above mentioned dosing regimen (Table 1) [13].

Observations on tolerance were based on 2360 administrations in 189 cases of whom data on liver enzymes [alanine transaminase (ALT), aspartate aminotransferase (AST) and gamma-glutamyl transferase $(\gamma \mathrm{GT})$, all U/L] were available in $149(79 \%)$ cases, resulting in 1132 (ALT $\mathrm{n}=$ 485 , AST $n=491$, and $\gamma \mathrm{GT} n=156$ ) liver enzymes quantifications. There were no significant differences in ALT or $\gamma \mathrm{GT}$ values when pre-treatment observations were compared with observations during or after treatment. Data were subsequently analysed in 3 predefined subgroups with a perceived increased risk to develop hepatic intolerance, i.e. in preterm neonates, in cases in whom iv administration exceeded 72 hours and finally, in cases in whom preadministration AST or ALT values exceeded 100 and 50 IU/L respectively [13].

Prematurity was documented in 62 cases. In this subgroup, median values of ALT $(\mathrm{n}=154)$, AST $(\mathrm{n}=155)$ and $\gamma \mathrm{GT}(\mathrm{n}=60)$ were $9(4-61), 24$ (8-198) and 92 (12-239) U/L respectively. There was no significant increase in any of the liver enzymes when observations before were compared with observations during and/or after iv administration. An inverse correlation of AST ( $\mathrm{r}=-0.26,95 \% \mathrm{CI}-0.44$ to 0.06 ) with duration of administration (days) was observed. Duration of iv administration exceeded 72 hours in 73 cases. In this subgroup, median values of ALT $(\mathrm{n}=270)$, AST $(\mathrm{n}=$ $274)$ and $\gamma \mathrm{GT}(\mathrm{n}=71)$ were $13(4-228), 29(8-389)$ and 53 (12 - 255) U/L respectively. There was no significant increase in any of the liver enzymes when observations before were compared with observations during and/or after iv administration of paracetamol. An inverse correlation of AST ( $\mathrm{r}=-0.18,95 \% \mathrm{CI}-0.32$ to -0.03$)$ with duration of administration (days) was observed. In 15 cases, a AST value $>100 \mathrm{U} / \mathrm{L}$ was documented before initiation of iv paracetamol. Median AST $(n=22)$ before iv paracetamol was 127 (53 - 1068). During iv administration, median AST (n =33) was $56(16-376)$ and following iv administration (n =11) $47(16-198) \mathrm{U} / \mathrm{L}$. In 8 cases, a ALT value $>50 \mathrm{U} / \mathrm{L}$ was documented before initiation of iv paracetamol. Median $\operatorname{ALT}(\mathrm{n}=14)$ before iv paracetamol was $98(36-422)$. During iv administration, median ALT $(\mathrm{n}=26)$ was $26(16-216)$ and following iv administration $(\mathrm{n}=11) 35(4-84) \mathrm{U} / \mathrm{L}$. It was therefore concluded that the absence of any increase of liver enzymes during and after administration of iv paracetamol in neonates strongly suggests that intravenous paracetamol is well tolerated in pre(term) neonates, in concordance with reports in children $[13,20]$.

\section{DISCUSSION}

More than one century after the introduction of paracetamol into clinical practice, still new observations on its disposition are unveiled and relevant questions can be formulated [6]. In a stepwise approach, we were able to describe aspects of disposition, metabolism and tolerance of intravenous paracetamol in preterm and term neonates $[4,5$, $12,13]$. Progressive maturation of paracetamol clearance in neonates, infants and toddlers is illustrated in Fig. (3), reflecting a lower clearance in early life. Based on the available pharmacokinetic observations, it seems that intravenous paracetamol is an attractive analgesic to be considered for use in neonates, potentially as a alternative for opioids or to reduce the need for moderate potent (e.g. tramadol) or potent opioids (morphine, fentanyl) in neonates [21].

Analgesia of paracetamol is mediated through inhibition of prostaglandins synthesis in the central nervous system (cyclo-oxygenase III and IIb). Analgesic effects also involve inhibitory action at the level of spinal nitric oxide and serotonergic pathways $[6,21]$. Paracetamol is believed to be an effective antipyretic at plasma concentrations between 10 and $20 \mathrm{mg} / \mathrm{L}$ and these concentrations have also been suggested to provide analgesia $[9,21]$. However, data on pharmacodynamics of intravenous paractamol in neonates are still lacking [1].

Further studies on the pharmacodynamics of this drug in this specific population are urgently needed similar to the recent work of Capici et al. on the pharmacodynamics of intravenous paracetamol in children after adenotonsillectomy [22]. These authors compared the time until rescue medication after adeno-tonsillectomy in children was needed after paracetamol administration. They hereby were able to document that a 6 hour interval of intravenous administration $(20 \mathrm{mg} / \mathrm{kg})$ should not be exceeded. The time until rescue medication was needed was shorter after intravenous administration $(6 \mathrm{~h})$ compared to rectal $(40 \mathrm{mg} / \mathrm{kg})$ administration $(10 \mathrm{~h})$, potentially in part reflecting the slower and more variable absorption after rectal administration. Since the time until rescue medication was the main outcome variable of this study, no final conclusions on the safety/effectiveness balance during repeated rectal or intravenous administration of paracetamol can be drawn based on this study. 
In contrast to the limited data in children, pharmacodynamic studies with repeated intravenous paracetamol administration in neonates are still completely absent. We hope that the recent European initiative to stimulate clinical studies in children and the establishment of research networks to perform these clinical trials will improve the feasibility to perform these type of studies in order to generate the evidence on which medicines are used in children [23]. Such an approach should result in an safer and more effective prescription and use of drugs in neonates. This even is true for frequently administered drugs like paracetamol since still important issues on its use remain to be unveiled.

In conclusion, disposition, metabolism and tolerance of intravenous paracetamol in neonates have been documented. In contrast to the available observations on the pharmacokinetics of this drug in neonates, we are unaware of any pharmacodynamic study (level of analgesia) on intravenous paracetamol in neonates. Prospective, well designed and appropriated powered studies are urgently needed.

\section{ACKNOWLEDGEMENT}

The clinical research of $\mathrm{K}$ Allegaert is supported by the Clinical Research Fund (Klinisch Onderzoeksfonds) of the University Hospitals Leuven, Belgium.

\section{REFERENCES}

[1] Tibboel D, Anand KJ, van den Anker JN. The pharmacological treatment of neonatal pain. Semin Fetal Neonatal Med 2005; 10: 195-205.

[2] Anderson BJ, van Lingen RA, Hansen TA, Lin Y-C, Holford NHG. Acetaminophen developmental pharmacokinetics in premature neonates and infants. Anesthesiology 2002; 96: 1336-45.

[3] Murat I, Baujard C, Foussat C, et al. Tolerance and analgesic efficacy of a new i.v. paracetamol solution in children after inguinal hernia repair. Paediatr Anaesth 2005; 15: 663-70.

[4] Allegaert K, Van der Marel CD, Pluim MAL, et al. Pharmacokinetics of single dose propacetamol in neonates: effect of gestational age. Arch Dis Child Fetal Neonat Ed 2004; 89: F25-8.

[5] Allegaert K, Anderson BJ, Naulaers G, et al. Intravenous paracetamol (propacetamol) pharmacokinetics in term and preterm neonates. Eur J Clin Pharmacol 2004; 60: 191-7.

[6] Prescott LF. Paracetamol. A critical bibliographic review. Second edition. London, Taylor and Francis Publishers, 2001.

[7] Miller RP, Roberts RJ, Fischer LJ. Acetaminophen elimination kinetics in neonates, children and adults. Clin Pharmacol Ther 1976; 19: 284-94.
[8] Van der Marel CD, Anderson BJ, Van Lingen RA, et al. Paracetamol and metabolite pharmacokinetics in infants. Eur J Clin Pharmacol 2003; 59: 243-51

[9] Anderson BJ, Woollard GA, Holford NH. Acetaminophen analgesia in children: placebo effect and pain resolution after tonsillectomy. Eur J Clin Pharmacol 2001; 57: 559-69.

[10] Allegaert K, Verbesselt R, Devlieger H, de Hoon J, Tibboel D. Cerebrospinal fluid pharmacokinetics of intravenous propacetamol in a former preterm infant. Br J Clin Pharmacol 2004; 57: 224-5.

[11] Kumpulainen E, Kokki H, Halonen T, Heikkinen M, Savolainen J, Laisalmi M. Paracetamol (acetaminophen) penetrates readily into the cerebrospinal fluid of children after intravenous administration. Pediatrics 2007; 119: 766-71.

[12] Allegaert K, de Hoon J, Verbesselt R, Vanhole C, Devlieger H, Tibboel D. Intra- and interindividual variability of glucuronidation of paracetamol during repeated administration in neonates. Acta Paediatr 2005; 94: 1273-9.

[13] Allegaert K, Rayyan M, de Rijdt T, van Beek F, Naulaers G. Hepatic tolerance of repeated intravenous paracetamol administration in neonates. Paediatr Anaesth 2008; 18: 388-92.

[14] Allegaert K, Murat I, Anderson B. Not all intravenous paracetamol formulations are created equal. Paediatr Anaesth 2007; 17: 811-2.

[15] Ghanem CI, Ruiz ML, Villanueva SS, et al. Shift from biliary to urinary elimination of acetaminophen glucuronide in acetaminophen pretreated rats. J Pharmacol Exp Ther 2005; 315: 987-95.

[16] Gelotte CK, Auiler JF, Lynch JM, Temple AR, Slattery JT. Disposition of acetaminophen at 4,6 , and $8 \mathrm{~g}$ /day for 3 days in healthy young adults. Clin Pharmacol Ther 2007; 81: 840-8.

[17] Kearns GL, Leeder JS, Wasserman GS. Acetaminophen overdose with therapeutic intent. J Pediatr 1998; 132: 5-8.

[18] Walls L, Baker CF, Sarkar S. Acetaminophen-induced hepatic failure with encephalopathy in a newborn. J Perinatol 2007; 27: 133-5.

[19] Kearns GL, Abdel-Rahman SM, Alander SW, Blowey DL, Leeder JS, Kaufman RE. Developmental pharmacology: drug disposition, action, and therapy in infants and children. N Engl J Med 2003; 349: 1157-67.

[20] Palmer GM, Chen SP, Smith KR, Hardikar W. Introduction and audit of intravenous paracetamol at a tertiary paediatric teaching hospital. Anaesth Intensive Care 2007; 35: 705-9.

[21] Anderson BJ, Gibb IA. Paracetamol (acetaminophen) pharmacodynamics : interpreting the plasma concentration. Arch Dis Child 2008; 93: 241-7.

[22] Capici F, Ingelmo PM, Davidson A, et al. Randomized controlled trial of duration of analgesia following intravenous or rectal acetaminophen after adenotonsillectomy in children. Br J Anaesth 2008; 100: 251-5.

[23] Choonara I. Improving children's medicines. Arch Dis Child 2006; 91: 550-1. 